\title{
Predictores robustos de estructura fija
}

\author{
D. Tena, I. Peñarrocha, R. Sanchis \\ Departament d'Enginyeria de Sistemes Industrials i Disseny \\ Universitat Jaume I de Castelló, España \\ \{david.tenatena,ipenarro,rsanchis\}@uji.es
}

\section{Resumen}

En este trabajo se propone el análisis y diseño de predictores robustos de estructura fija para su uso en el control de procesos con retardos puros, como alternativa al predictor de Smith y al observador de estados. Respecto al primero, se demuestra mejorar la robustez ante incertidumbre del modelo. Respecto al segundo, se reduce ampliamente el número de parámetros de ajuste, facilitando su implementación en plataformas industriales. Para el diseño, se plantea la minimización de varias normas del sistema mediante un problema de optimización con desigualdades matriciales y se obtienen los parámetros del predictor utilizando algoritmos de optimización. Finalmente, se desarrolla un ejemplo numérico de aplicación práctica.

Palabras clave: predictores, estructura fija, predictor de Smith, procesos con retardo, robustez.

\section{INTRODUCCIÓN}

Los retardos puros suponen un reto para el control de los procesos en los que aparecen [7]. Los problemas asociados a retardos implican una reducción de las prestaciones del control así como de la robustez de los sistemas en bucle cerrado. Se han desarrollado técnicas basadas en la estimación o predicción del valor futuro de la salida de modo que se elimine el retardo en la realimentación y se puedan sintonizar los controladores como si el proceso careciese del retardo. En general, estas técnicas toman la señal de entrada al proceso y la señal de salida y las procesan para estimar el valor futuro de la salida.

La técnica más popular para predecir la salida futura es el predictor de Smith [8]. El predictor de Smith cancela completamente el retardo y permite sintonizar el controlador teniendo en cuenta únicamente la función de transferencia del proceso sin retardo. El principal problema que presenta es su poca robustez ante incertidumbre del modelo, en especial ante incertidumbre en el valor del retardo. Esto hace que su aplicación industrial esté limitada y requiera de modificaciones sobre la estructura principal [7].
Otra solución más compleja consiste en la utilización de un observador del estado y un controlador de realimentación del estado observado en lugar de uno de realimentación de la salida [5]. Esta alternativa también tiene algunos inconvenientes. Por un lado, su implementación es complicada en sistemas de control industriales como PLC's o Sistemas de Control Distribuido, pues requiere la configuración de multitud de parámetros en la programación del algoritmo debido al gran tamaño de las matrices que representan el modelo. Por otro lado, la sintonización del observador requiere de un conocimiento técnico elevado que suele quedar fuera del alcance del usuario medio, por ejemplo un operador de producción en una planta industrial.

Por tanto, en este trabajo trataremos de sintetizar un predictor que lidie con los inconvenientes de ambas alternativas. Tendremos como requisitos que sea robusto ante incertidumbre del modelo y que conste de estructura fija y pocos parámetros de ajuste de modo que sea intuitivo y fácil de implementar en plataformas industriales.

En el apartado 2 se describe el problema de predicción y cómo lo afronta el predictor de Smith. En el apartado 3 se justifica la estructura del predictor propuesto y se explica la técnica para su diseño y el análisis de robustez. En el apartado 4 se demuestran los resultados a través de un ejemplo. El apartado final resume las conclusiones del trabajo.

\section{DESCRIPCIÓN DEL PROBLEMA}

En la figura 1 se muestra la estructura de control genérica de un predictor de la salida para controlar un proceso con retardo puro.

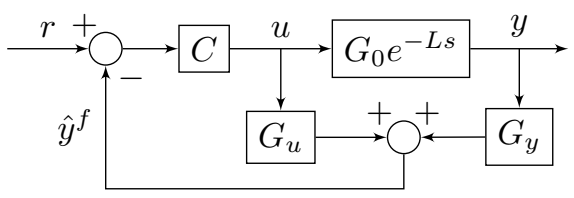

Figura 1: Estructura en bucle cerrado con predictor de la salida. 
Sea $G(s)$ un sistema monovariable FOTD (sistema de primer orden con retardo),

$$
G(s)=\frac{k}{(1+T s)} e^{-L s},
$$

para que la señal $\hat{y}^{f}=G_{u} u+G_{y}$ y sea la predicción exacta de la salida $y$ en el tiempo futuro $t=L$, se debe cumplir

$$
\begin{array}{r}
G_{u}(s)+G_{0}(s) e^{-L s} G_{y}(s)=G_{0}(s) \Rightarrow \\
\quad \Rightarrow G_{u}(s)=G_{0}(s)\left(1-e^{-L s} G_{y}(s)\right) .
\end{array}
$$

El predictor de Smith cumple con esto ya que puede expresarse como un caso particular de la estructura de la figura 1 donde

$$
G_{u}(s)=G_{0}(s)\left(1-e^{-L s}\right), G_{y}(s)=1 .
$$

La figura 2 muestra las respuestas ante entrada escalón de $G_{u}$ y $G_{y}$ para el predictor de Smith según (3). En este ejemplo $G(s)$ tiene $k=1, T=5$, $L=25$. Como se puede observar, el comportamiento consiste en predecir la salida mediante $u$ durante el tiempo $t<L$ y en sustituir la predicción por la salida $y$ a partir de $t>L$.
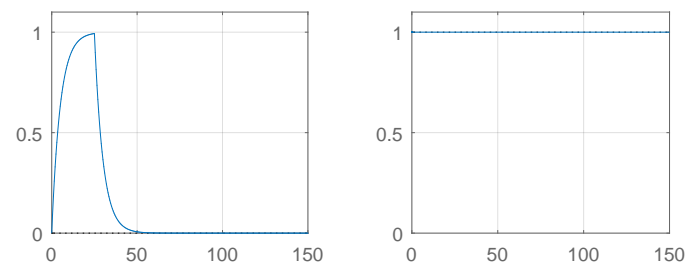

Figura 2: Respuesta ante escalón de $G_{u}(s)$ (izquierda) y $G_{y}(s)$ (derecha) para predictor de Smith.

El problema que se plantea es el de diseñar un predictor que aproxime las dinámicas de $G_{u}$ y $G_{y}$ descritas y sea robusto ante cambios del modelo. Cabe reflexionar que hay que tener especial cuidado en aproximar bien $G_{u} u$ en $t<L$, puesto que en ese periodo sólo se conoce el valor de $u$ y se predice en bucle abierto. En $t>L$, se puede hacer uso de la señal $y$. Nótese que según (3) el error de predicción se anula en estado estacionario gracias a que $G_{u}(0)=0$ y $G_{y}(0)=1$.

\section{DISEÑO DEL PREDICTOR PROPUESTO}

\subsection{Estructura del predictor}

La estructura del predictor debe cumplir los requisitos descritos en el apartado anterior. Se decide que $G_{u}$ tenga un derivador (cero en $s=0$ ) para cumplir que su respuesta ante escalón sea 0 en estado estacionario. Se añaden dos polos por ser el número mínimo de polos que permite que $G_{u}$ sea realizable dado que contiene un derivador. La ganancia estática de $G_{y}$ será 1 para que en estado estacionario la salida del predictor coincida con $y$. Además añadimos un polo en $G_{y}$ ya que la estructura de $G_{u}$ no aproxima bien la parte descendente de la respuesta con predictor de Smith, por tanto necesitamos añadir una dinámica más lenta en $G_{y}$ para compensar. Con estos criterios la estructura del predictor propuesto consta de

$$
G_{u}(s)=\frac{k_{u} s}{\left(1+\tau_{u} s\right)^{2}}, G_{y}(s)=\frac{1}{\left(1+\tau_{y} s\right)} .
$$

De este modo podemos aproximar la respuesta del sistema con predictor de Smith, como se puede ver en la figuras 3 y 4 , con una estructura fija y utilizando únicamente tres parámetros de ajuste: $k_{u}, \tau_{u}$ y $\tau_{y}$. En las figuras, $k_{u}=50, \tau_{u}=18 \mathrm{y}$ $\tau_{y}=25$. En la figura 5 se muestra el diagrama de Bode en magnitud y frecuencia del sistema con el predictor de Smith y el predictor propuesto.
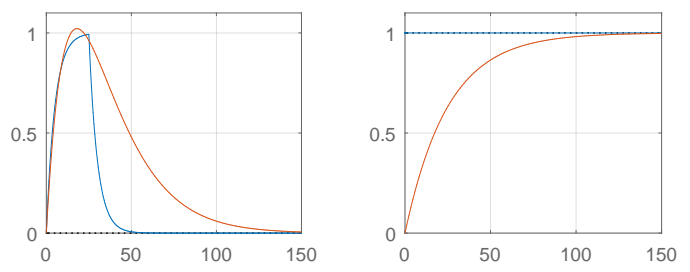

Figura 3: Respuesta ante escalón de $G_{u}(s)$ (izquierda) y $G_{y}(s)$ (derecha) para predictor de Smith (azul) y predictor propuesto (rojo).

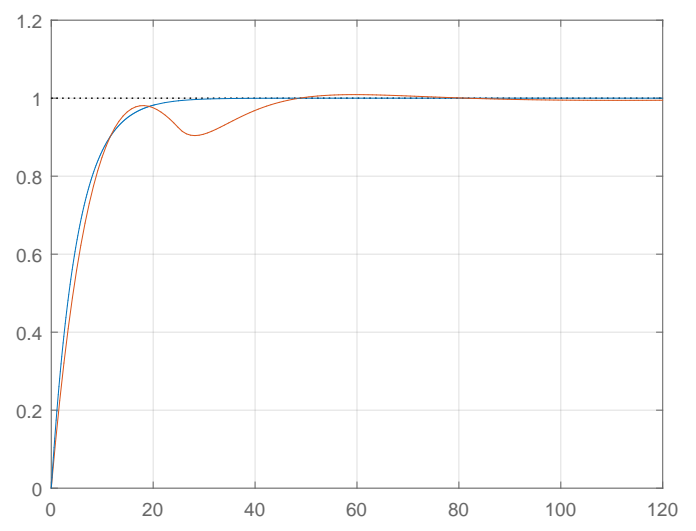

Figura 4: Respuesta de $\hat{y}^{f}$ ante escalón de $u$ para predictor de Smith (azul) y predictor propuesto (rojo).

\subsection{Diseño óptimo del predictor}

Sea $G(s)$ el sistema descrito en (1) en espacio de estados definido por las matrices $a, b$ y $c$; y sea 


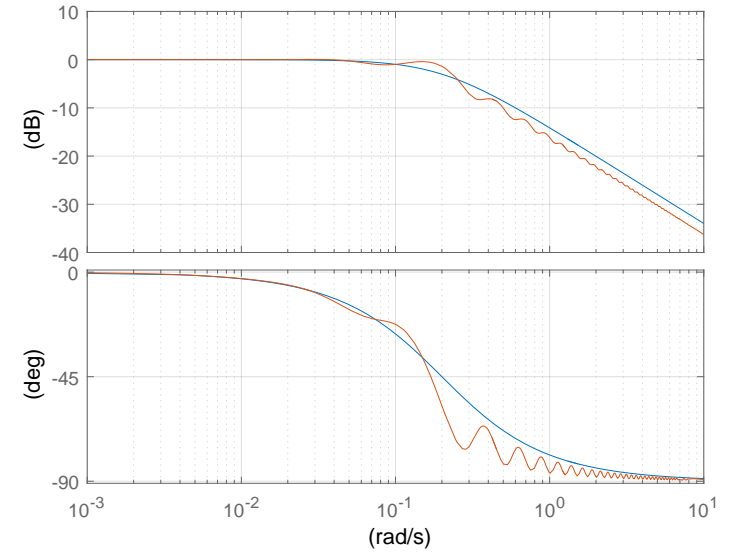

Figura 5: Diagrama de Bode del sistema con predictor de Smith (azul) y predictor propuesto (rojo).

$G_{p}(s)$ la aproximación de Padé del sistema definida por $a_{p}, b_{p}, c_{p} \mathrm{y} d_{p}$ [7]; la realización en espacio de estados de la aproximación de Padé del sistema con retardo es

$$
\begin{aligned}
\dot{x} & =A x+B u \\
y & =C x \\
y^{f} & =C_{f} x
\end{aligned}
$$

donde

$$
\begin{array}{ll}
A=\left[\begin{array}{cc}
a & 0 \\
b_{p} c & a_{p}
\end{array}\right], \quad B=\left[\begin{array}{l}
b \\
0
\end{array}\right], \\
C=\left[\begin{array}{ll}
d_{p} c & c_{p}
\end{array}\right] \quad, \quad C_{f}=\left[\begin{array}{ll}
c & 0
\end{array}\right] .
\end{array}
$$

Los mejores resultados han sido obtenidos con aproximaciones de Padé de orden 2. Nótese que $y^{f}$ es el valor futuro de $y$ en $t=L$.

El predictor propuesto se puede escribir como un observador de la salida $[2,3]$,

$$
\begin{aligned}
\dot{\xi} & =P \xi+Q u+R y \\
\hat{y}^{f} & =S \xi+T u+V y
\end{aligned}
$$

donde, para cumplir con la estructura descrita en (4) y empleando la realización canónica observable,

$$
\begin{gathered}
P=\left[\begin{array}{ccc}
0 & -p_{u}^{2} & 0 \\
1 & -2 p_{u} & 0 \\
0 & 0 & -p_{y}
\end{array}\right], Q=\left[\begin{array}{c}
0 \\
K_{u} \\
0
\end{array}\right], \\
R=\left[\begin{array}{c}
0 \\
0 \\
p_{y}
\end{array}\right], S=\left[\begin{array}{l}
0 \\
1 \\
1
\end{array}\right] T=0, V=0,
\end{gathered}
$$

siendo

$$
K_{u}=\frac{k_{u}}{\tau_{u}^{2}}, p_{u}=\frac{1}{\tau_{u}}, p_{y}=\frac{1}{\tau_{y}} .
$$

Definimos el error de predicción como $y^{f}-\hat{y}^{f}$. Se diseñará el predictor de modo que la norma $\mathcal{H}_{\infty}$ o $\mathcal{H}_{2}$ del error de predicción sea mínima ante cambios en $u$.

El sistema extendido que tiene por salida el error de predicción es

$$
\begin{aligned}
{\left[\begin{array}{c}
\dot{x} \\
\dot{\xi}
\end{array}\right] } & =\mathcal{A}\left[\begin{array}{l}
x \\
\xi
\end{array}\right]+\mathcal{B} u \\
{\left[y^{f}-\hat{y}^{f}\right] } & =\mathcal{C}\left[\begin{array}{l}
x \\
\xi
\end{array}\right]
\end{aligned}
$$

donde

$$
\begin{aligned}
\mathcal{A} & =\left[\begin{array}{cc}
A & \mathbf{0} \\
R C & P
\end{array}\right] \quad, \quad \mathcal{B}=\left[\begin{array}{l}
B \\
Q
\end{array}\right], \\
\mathcal{C} & =\left[\begin{array}{ll}
C & -S
\end{array}\right] .
\end{aligned}
$$

El problema de optimización queda definido por

$$
\begin{array}{rc}
\min & \gamma \\
\text { s.a } & \Psi<0
\end{array}
$$

donde $\Psi$ representa una desigualdad matricial bilineal (BMI) que, según la norma que se desee minimizar, será

$$
\begin{array}{r}
\Psi_{\mathcal{H}_{\infty}}:\left[\begin{array}{ccc}
\mathcal{A} Z+Z \mathcal{A}+\mathcal{C}^{T} \mathcal{C} & Z \mathcal{B} \\
\star & & -\gamma I
\end{array}\right], \\
\Psi_{\mathcal{H}_{2}}:\left[\begin{array}{ccc}
\mathcal{A} Z+Z \mathcal{A}+\mathcal{C}^{T} \mathcal{C} & 0 & 0 \\
\star & -\gamma I & \mathcal{B}^{T} Z \\
\star & \star & -Z
\end{array}\right],
\end{array}
$$

siendo $Z$ una matriz simétrica semidefinida positiva [1]. Según la norma utilizada, el criterio de diseño es el siguiente:

$$
\begin{aligned}
\mathcal{H}_{\infty} & :\left\|y^{f}-\hat{y}^{f}\right\|_{2}<\gamma\|u\|_{2} \\
\mathcal{H}_{2} & :\left\|y^{f}-\hat{y}^{f}\right\|_{R M S}<\gamma \sigma_{u} .
\end{aligned}
$$

Esta técnica de diseño es válida sólo para sistemas estables debido a la estructura triangular de $\mathcal{A}$, que contiene en el primer elemento la matriz $A$ de dinámica del sistema. Si $A$ contiene polos inestables, el predictor resultante será inestable también.

Para la resolución del problema de optimización con BMIs y la obtención de los parámetros del predictor se recomienda el uso de la herramienta penbmi [6].

\subsection{Robustez del predictor}

Para el análisis de robustez se parte de la estructura de control definida en la figura 1. La función de sensibilidad del sistema en bucle cerrado viene dada por

$$
\mathcal{S}=\frac{1}{1+G_{0} e^{-L s} G_{y \frac{C}{1+C G_{u}}}} .
$$


Se define el margen de sensibilidad como

$$
M_{s}=\operatorname{máx}_{\omega}|\mathcal{S}(j \omega)| .
$$

Según $[4,9]$, los valores razonables de $M_{s}$ están entre 1,2 y 2 , y corresponden a márgenes de ganancia de entre 6 y 2 y a márgenes de fase de entre $49^{\circ}$ y $29^{\circ}$ para el sistema en bucle abierto.

\section{EJEMPLO}

Consideremos un sistema FOTD según (1) con $k=1, T=5$ y $L=25$. El controlador $C$ será un controlador PI con los parámetros de ajuste

$$
K_{p}=\frac{1}{k}, T_{i}=\frac{T}{3} .
$$

Con esto aseguramos un margen de fase de $60^{\circ}$ para un sistema de primer orden [5]. El controlador recibirá la señal de error entre la referencia $r$ y la salida futura estimada $\hat{y}^{f}$. Las figuras 6 y 7 muestran la respuesta del sistema en bucle cerrado ante cambio de referencia escalón y ante perturbación de entrada escalón, respectivamente, para el predictor de Smith y el predictor propuesto con diferentes criterios de diseño. En las mismas figuras se pueden ver también estas respuestas si el retardo del proceso cambia de 25 a 21 unidades, lo que ilustra cómo el sistema con predictor de Smith se vuelve inestable.
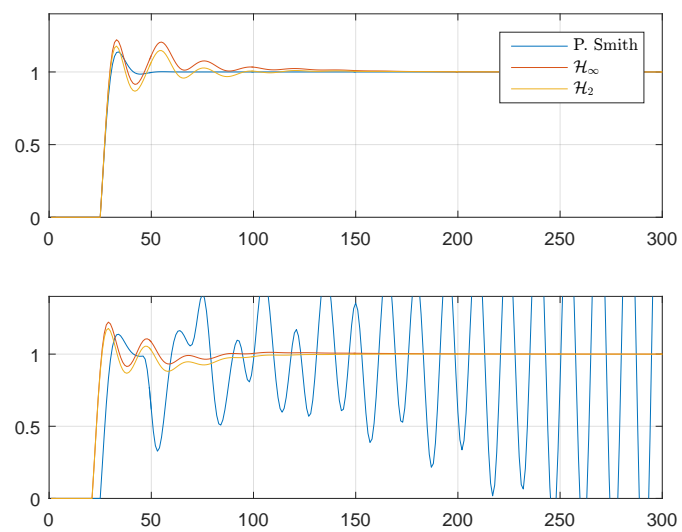

Figura 6: Respuesta ante cambio de referencia escalón sin cambio del retardo (arriba) y con cambio del retardo (abajo).

Debido al carácter oscilatorio que presenta la respuesta ante escalón de referencia del predictor propuesto, se recomienda la aplicación de un filtro para la referencia con una constante de tiempo del orden de $L / 4$. En la figura 8 se puede ver la respuesta ante escalón de referencia tras aplicar un filtro de este modo.
Los parámetros del predictor propuesto, hallados mediante la técnica comentada en el apartado anterior, se detallan en el cuadro 1.

Cuadro 1: Parámetros de ajuste óptimos del predictor propuesto.

\begin{tabular}{lccc}
\hline & $k_{u}$ & $\tau_{u}$ & $\tau_{y}$ \\
\hline $\mathcal{H}_{\infty}$ & 45,98 & 17,27 & 25,63 \\
$\mathcal{H}_{2}$ & 48,58 & 17,43 & 24,22 \\
\hline
\end{tabular}
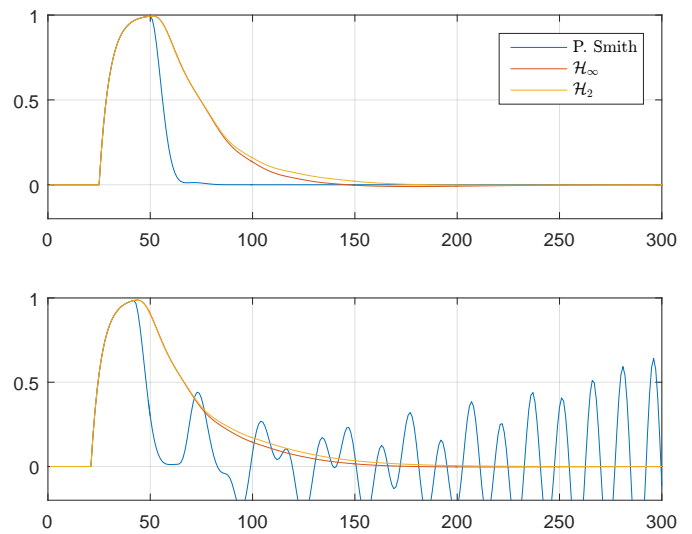

Figura 7: Respuesta ante perturbación de entrada escalón sin cambio del retardo (arriba) y con cambio del retardo (abajo).
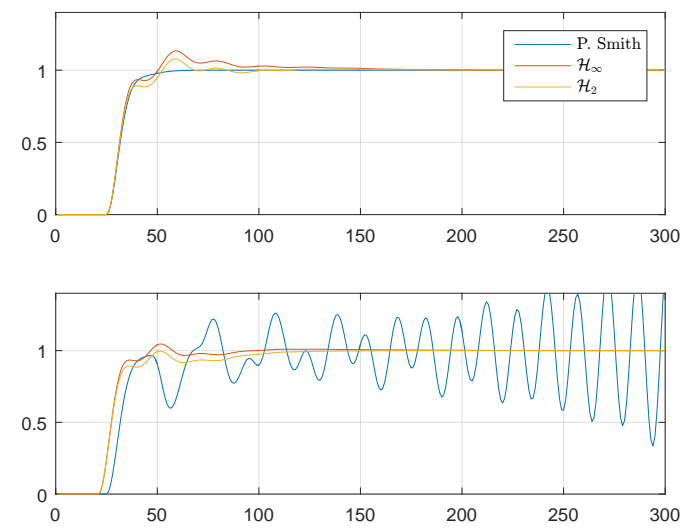

Figura 8: Respuesta ante cambio de referencia filtrada sin cambio del retardo (arriba) y con cambio del retardo (abajo).

Para la comparativa de robustez de ambos predictores, se muestra en la figura 10 el diagrama de Nyquist del sistema con predictor de Smith y con el predictor propuesto. Se observa cómo el predictor de Smith reduce el margen de fase y de ganancia frente al predictor propuesto. En la figura 11 comprobamos cómo el sistema se vuelve inestable con predictor de Smith si el retardo del proceso cambia de 25 a 21 unidades, mientras que el diagrama de Nyquist del sistema con los predictores propuestos apenas varía. 

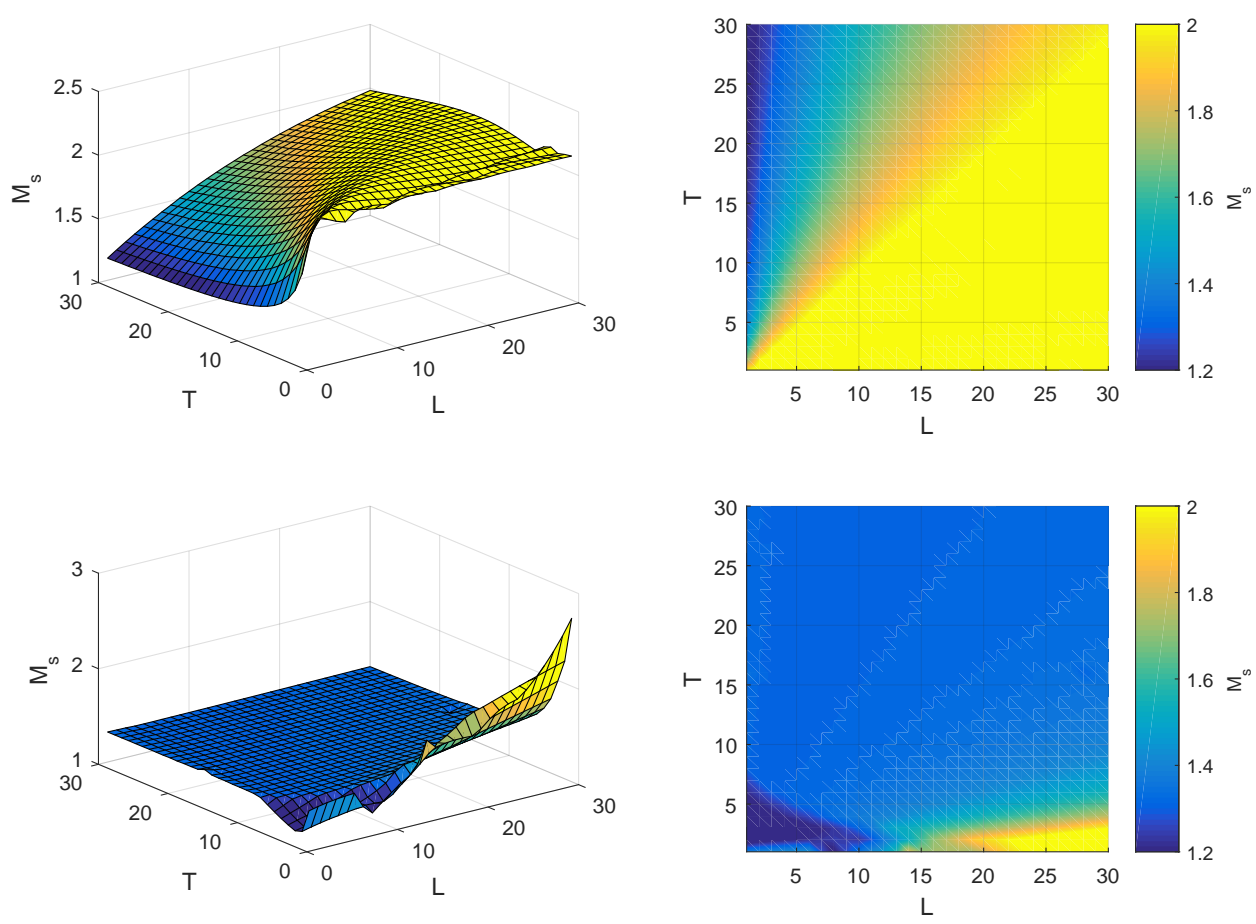

Figura 9: Margen de sensibilidad con el predictor de Smith (arriba) y el predictor propuesto $\mathcal{H}_{\infty}$ (abajo).

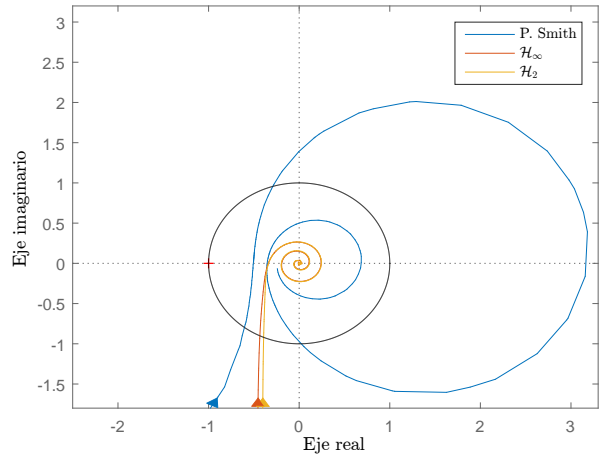

Figura 10: Diagrama de Nyquist del sistema con predictor de Smith y con el predictor propuesto sin cambio del retardo.

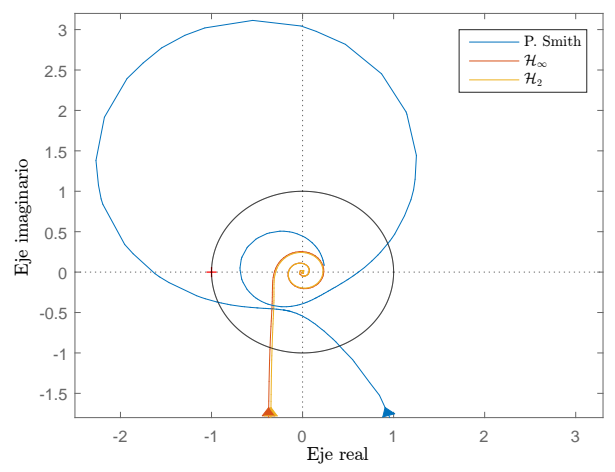

Figura 11: Diagrama de Nyquist del sistema con predictor de Smith y con el predictor propuesto con cambio del retardo.
Para relacionar la robustez con la dinámica original del sistema (1), se ha calculado también el margen de sensibilidad $M_{s}$ del sistema en bucle cerrado para un rango de dinámicas diferentes, siendo $k=1, T \in[1,30]$ y $L \in[1,30]$. La figura 9 muestra los resultados.

Nótese que para valores de $T<<L$ y $T>>L$, el predictor propuesto presenta problemas numéricos de cálculo en el algoritmo de optimización por mal condicionamiento de la función de transferencia del sistema.

Se observa como con el predictor de Smith alcanzamos valores de $M_{s}>2$ aproximadamente cuando $\frac{L}{T}>1$, mientras que con el predictor propuesto se cumple con los criterios de robustez en prácticamente todo el rango de valores.

\section{CONCLUSIONES}

Se ha propuesto el análisis y diseño de predictores robustos de estructura fija para su uso en el control de procesos con retardos puros, como alternativa al predictor de Smith y al observador de estados. Se ha demostrado una mejoría en la robustez ante incertidumbre del modelo respecto al primero. Respecto al segundo, se ha conseguido reducir el número de parámetros de ajuste a tan sólo tres, facilitando así su implementación en plataformas industriales. Se han utilizado algoritmos de optimización para resolver el problema con desigualdades matriciales relacionado con 
la minimización de varias normas del sistema. Finalmente, se ha desarrollado un ejemplo numérico de aplicación práctica, mostrando la bondad de la propuesta.

\section{Agradecimientos}

Este trabajo ha sido financiado por la Universitat Jaume I de Castelló a través del proyecto con referencia P1·1B2015-42 y por el Ministerio de Economía y Competitividad a través del proyecto con referencia TEC2015-69155-R.

\section{Referencias}

[1] Stephen P Boyd, Laurent El Ghaoui, Eric Feron, and Venkataramanan Balakrishnan. Linear matrix inequalities in system and control theory, volume 15. SIAM, 1994.

[2] Jie Chen and Ron J Patton. Robust modelbased fault diagnosis for dynamic systems, volume 3. Springer Science \& Business Media, 2012.

[3] Steven Ding. Model-based fault diagnosis techniques: design schemes, algorithms, and tools. Springer Science \& Business Media, 2008.

[4] Olof Garpinger, Tore Hägglund, and Karl Johan Åström. Performance and robustness trade-offs in pid control. Journal of Process Control, 24(5):568-577, 2014.

[5] Graham Clifford Goodwin, Stefan F Graebe, and Mario E Salgado. Control system design, volume 240. Prentice Hall New Jersey, 2001.

[6] Michal Kocvara and Michael Stingl. Penbmi user's guide. Avaiable from http://www. penopt. com, 2005.

[7] Julio E Normey-Rico and Eduardo F. Camacho. Control of dead-time processes. Springer Science \& Business Media, 2007.

[8] ZJ Palmor. Time-delay compensation - smith predictor and its modifications. The control handbook, 1:224-229, 1996.

[9] Sigurd Skogestad and Ian Postlethwaite. Multivariable feedback control: analysis and design, volume 2. Wiley New York, 2007. 